\section{BRAZIULIAN JOURNAL \\ OF MEDICAL AND BIOLOGICAL RESF.ARCH}

www.bjournal.com.br
ISSN 0100-879X

Volume 43 (04) 325-408

April 2010

BIOMEDICAL SCIENCES

AND

CLINICAL INVESTIGATION

Braz J Med Biol Res, March 2010, Volume 43(4) 397-402

Immediate effects of submaximal effort on pulse wave velocity in patients with Marfan syndrome

P. Peres, G.F. Bernardelli, C.C. Mendes, S.S.C.P.M. Fischer, D.M. Servantes, W.M. Medeiros and A.C. Carvalho

The Brazilian Journal of Medical and Biological Research is partially financed by
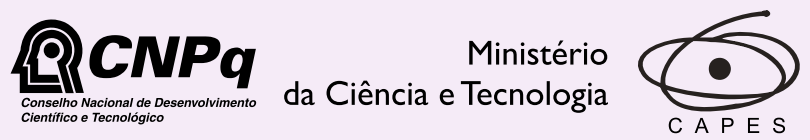

Ministério da Educação

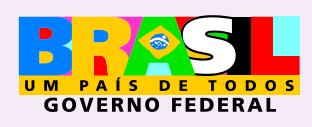

Institutional Sponsors 


\title{
Immediate effects of submaximal effort on pulse wave velocity in patients with Marfan syndrome
}

\author{
P. Peres, G.F. Bernardelli, C.C. Mendes, S.S.C.P.M. Fischer, \\ D.M. Servantes, W.M. Medeiros and A.C. Carvalho \\ Disciplina de Cardiologia, Universidade Federal de São Paulo, São Paulo, SP, Brasil
}

\begin{abstract}
Marfan syndrome (MS) is a dominant autosomal disease caused by mutations in chromosome 15, the locus controlling fibrillin 1 synthesis, and may exhibit skeletal, ocular, cardiovascular, and other manifestations. Pulse wave velocity (PWV) is used to measure arterial elasticity and stiffness and is related to the elastic properties of the vascular wall. Since the practice of exercise is limited in MS patients, it was of interest to analyze the acute effect of submaximal exercise on aortic distensibility using PWV and other hemodynamic variables in patients with MS with either mild or no aortic dilatation. PWV and physiological variables were evaluated before and after submaximal exercise in 33 patients with MS and 18 controls. PWV was $8.51 \pm 0.58$ at rest and $9.10 \pm 0.63 \mathrm{~m} / \mathrm{s}$ at the end of exercise $(P=0.002)$ in the group with MS and $8.07 \pm 0.35$ and $8.98 \pm 0.56 \mathrm{~m} / \mathrm{s}$ in the control group, respectively $(P=0.004)$. Comparative group analysis regarding $P W V$ at rest and at the end of exercise revealed no statistically significant differences. The same was true for the group that used $\beta$-blockers and the one that did not. The final heart rate was $10 \%$ higher in the control group than in the MS group $(P=0.01)$. Final systolic arterial pressure was higher in the control group $(P=0.02)$. PWV in MS patients with mild or no aortic dilatation did not differ from the control group after submaximal effort.
\end{abstract}

Key words: Exercise; Aortic elasticity; Pulse wave velocity; Marfan syndrome

\section{Introduction}

Marfan syndrome is a dominant autosomal disease caused by mutations in chromosome 15, the locus controlling fibrillin 1 synthesis, and may exhibit skeletal, ocular, cardiovascular, skin, neurological, and pulmonary manifestations; patients often have a family history of the condition (1). Cardiovascular complications in Marfan syndrome may include mitral valve prolapse and regurgitation, dilatation of the pulmonary artery and mainly dilatation of the root of the aorta, which is the major cause of death in individuals affected by the syndrome (2). Pulse wave velocity (PWV) is used as a measure of arterial elasticity and stiffness and is related to the elastic properties of the vascular wall (3). A number of studies have demonstrated a variation in arterial elasticity in patients with isolated systolic hypertension and post-acute myocardial infarction $(4,5)$. A reduction in aortic distensibility, as measured by PWV in patients with Marfan syndrome, has been reported $(6,7)$.

The 2005 Guidelines of the 36th Bethesda Conference in the field of sports cardiology recommend that patients with Marfan syndrome and aortic dilatation greater than 40 $\mathrm{mm}$ (in adults) only play sports such as golf, billiards and bowling, as these sports offer no potential risk of bodily collisions $(8,9)$. As the practice of exercise is treated with caution in this population and there are practically no studies on this issue, it was of interest to analyze the acute effect of submaximal exercise on aortic distensibility using PWV and hemodynamic parameters in patients with Marfan syndrome with either mild or no aortic dilatation.

\section{Patients and Methods}

A controlled, cross-sectional study was carried out involving 39 patients with Marfan syndrome evaluated between August and September 2006. All patients were in treatment at the clinics of the Cardiology and Genetics sectors of Universidade Federal de São Paulo (SP, Brazil). Six patients were under 12 years of age and were excluded from the study. Thirty-three patients (17 men) fulfilled the eligibility

Correspondence: P. Peres, Disciplina de Cardiologia, UNIFESP, Rua Napoleão de Barros, 715, 10andar, 04024-002 São Paulo, SP, Brasil. Fax: +55-11-5571-0047. E-mail: pp.peres@uol.com.br

Received July 1, 2009. Accepted February 26, 2010. Available online March 19, 2010. Published April 12, 2010. 
criteria for participation in the study. The eligibility criteria required patients to have either a normal aorta or minimal dilatation (less than $37 \mathrm{~mm}$, as determined by an echocardiogram), with no prior dissection, a recent echocardiogram (previous 6 months), the absence of signs and symptoms of cardiac ischemia, neurological or motor disorders, and not having undergone heart surgery. Echocardiogram measures were obtained on the root of the aorta (Valsalva sinus) and were carried out by a single examiner not involved in the study, using the methodology and reference values of the Echocardiography Sector, Cardiology Division, UNIFESP, and previous studies $(10,11)$.

Among the 33 patients, 16 used $\beta$-blockers (propranolol or atenolol) and one used a $\beta$-blocker and angiotensin receptor antagonist (losartan). A group of 18 healthy individuals (9 men) paired for gender, age, and weight was selected as the control group. The study was approved by the Ethics Committee of the institution. All participants received information on the procedures to be carried out and signed a term of informed consent, which was also approved by the Ethics Committee for the present study.

\section{Pulse wave velocity}

PWV was determined by means of a noninvasive analysis of the propagation time and distance of the pulse wave between two acquisition points [PWV $=$ distance $(\mathrm{m}) /$ time (s)]. The transducers were positioned over the carotid and femoral arteries, always on the right side of the body and the signals were sent to the Complior ${ }^{\circledR}$ system (France) (12). Signal acquisition was performed by a skilled observer who was unaware of the patient's condition $(5,13)$. The measurements were obtained with the patient in a lying position before and after exertion on a stationary bike, with 15 sequential pulse waves preferentially recorded. The individual returned to the evaluation bed for the acquisition of the data as soon as the predicted heart rate $(\mathrm{HR})$ was reached or other exercise interruption criteria were fulfilled.

\section{Exercise protocol}

The individuals were submitted to an incremental test

Table 1. Anthropometric and echocardiographic data of patients with Marfan syndrome and controls.

\begin{tabular}{lcc}
\hline & Marfan $(\mathrm{N}=33)$ & Control $(\mathrm{N}=18)$ \\
\hline Age (years) & $19 \pm 8$ & $20 \pm 5$ \\
Weight $(\mathrm{kg})$ & $60.9 \pm 12.6$ & $62.4 \pm 11.9$ \\
Height $(\mathrm{m})$ & $1.78 \pm 0.10^{*}$ & $1.71 \pm 0.10$ \\
$\mathrm{BMI}\left(\mathrm{kg} / \mathrm{m}^{2}\right)$ & $19.32 \pm 3.93$ & $21.28 \pm 2.63$ \\
$\mathrm{ECHO}(\mathrm{mm})$ & $33 \pm 7^{*}$ & $28 \pm 2$ \\
\hline
\end{tabular}

Data are reported as means $\pm \mathrm{SD}$. BMI = body mass index; $\mathrm{ECHO}=$ echocardiogram. ${ }^{*} \mathrm{P} \leq 0.05$ compared to control group (t-test). limited by $H R$, which was calculated by the equation: $\mathrm{HR}_{\text {submax }}=195$ - age. If the patient was taking $\beta$-blockers, the calculated value was adjusted by the formula: \%HR to correct $=y+95.58 / 9.74$, in which $y$ is the daily amount of medication (in milligrams) the patient takes (14). The exercise was performed on a stationary bike (Queens ${ }^{\circledR}$ - QH 1000 , Brazil), with progressive increments in intensity every 2 min using the load control of the device. The participants were instructed to maintain a speed of 60 revolutions per minute. The exercise was interrupted when the participant reached the pre-calculated HR or manifested any signs or symptoms of exhaustion, dyspnea, fatigue, myocardial ischemia, blood pressure equal to or above $160 \times 100$ $\mathrm{mmHg}$ (15), or reached $14 \mathrm{~min}$ of exercise.

\section{Hemodynamic variables and blood lactate}

Physiological variables, HR and blood pressure were recorded after the patient remained at rest in a lying position for $5 \mathrm{~min}$, when seated prior to beginning the exercise, at the time of interruption, and after 1 min of recovery. HR was obtained using a heart monitor (Polar ${ }^{\circledR}$ - S 810, Finland) and blood pressure was determined using the auscultation method with a sphygmomanometer and stethoscope (Tycos ${ }^{\circledR}$, USA) (16). Blood lactate was determined from a blood sample obtained by puncture of the ring finger on the right hand while at rest, in the sitting position and at the time of interruption of exercise, using a lactometer (Accutrend ${ }^{\circledR}$ Lactate, Roche, USA).

\section{Statistical analysis}

The values of the variables considered for the analysis of HR, blood pressure and blood lactate were obtained with the individuals seated on the stationary bike at pre-test rest or at the time of interruption of exercise. Pulse wave time and velocity were obtained with the individuals lying down. Descriptive analysis is reported as the mean $\pm S D$ in absolute and percent values and presented in the tables.

The Kolmogorov-Smirnov test was employed in order to determine the normality of the data. Homogeneity of the sample was determined using the Levine test. For the analysis of the influence of systolic arterial pressure on the behavior of PWV, analysis of covariance (ANCOVA) was used, followed by the Bonferroni post hoc test when necessary. A paired Student $t$-test was used for intragroup inferential analysis and an independent Student $t$-test was used for intergroup analysis. A P value $\leq 0.05$ was considered to be significant.

\section{Results}

Table 1 presents the anthropometric data and echocardiogram measurements at the root of the aorta. The anthropometric analysis only revealed a significant difference in height between the two groups $(P=0.02$; Table 1$)$.

Seventeen $(51 \%)$ patients in the group with Marfan 
syndrome were taking $\beta$-blockers and their predicted HR were corrected by the previously described formula. Of the 33 patients, $14(42 \%)$ interrupted the exertion test by having reached the predicted HR, 8 due to peripheral limitation (leg fatigue), 7 for having reached the predetermined blood pressure level and 4 due to respiratory exhaustion (dyspnea). In the control group, 11 participants (61\%) interrupted the test for having reached the predicted HR, 4 for having reached the limit of $14 \mathrm{~min}$ of exercise, 1 due to peripheral limitation (leg fatigue), 1 due to dyspnea, and 1 test was interrupted when the participant's blood pressure reached 200 × $80 \mathrm{mmHg}$.

\section{Pulse wave propagation time and velocity}

The analysis of the propagation time and velocity of the pulse wave revealed different values at rest and at the end of exercise in both the patients with Marfan syndrome and the control group. The patients with Marfan syndrome had a PWV of $8.51 \pm 0.58$ at rest and $9.10 \pm 0.63 \mathrm{~m} / \mathrm{s}$ at the end of exercise $(P=0.002)$, while the pulse wave propagation time in this group was $78.27 \pm 4.98$ at rest and $73.48 \pm 5.57$ $\mathrm{ms}$ at the end of exercise $(P=0.015)$. In the control group, PWV was $8.07 \pm 0.35$ at rest and $8.98 \pm 0.56 \mathrm{~m} / \mathrm{s}$ at the end of the exercise $(P=0.004)$, while the propagation time was $79.22 \pm 3.93$ at rest and $71.50 \pm 4.76 \mathrm{~ms}$ at the end of exercise ( $P=0.012)$. Adjustment of PWV together with the final systolic arterial pressure revealed variation in this variable when the patients were at rest and at the end of exertion similar to the findings for PWV alone (ANCOVA; $P=0.0001$; Figure 1).

Comparative analysis of PWV revealed no significant differences between groups while at rest or at the end of exercise (ANCOVA). Similarly, there were no significant differences in pulse wave propagation time between groups at rest and at the end of exercise.

\section{Heart rate, blood lactate and exercise time}

The predicted HR was calculated for all individuals. The mean value reached by the patients with Marfan syndrome and the control group was $90 \%$ of the predicted HR ( $P=$ $0.44)$. There were no significant differences between groups regarding $H R$ values while at rest. Final $H R$ values were $10 \%$ higher in the control group than in the individuals with Marfan syndrome $(P=0.01)$. There were no significant differences between groups regarding HR values after $1 \mathrm{~min}$ of recovery (Table 2). There were significant differences between the control group and patients using $\beta$-blockers regarding $H R$ values while at rest $(P=0.04)$, at the end of the exercise $(P=0.01)$ and during the recovery phase $(P$ $=0.02)$. As expected, no significant differences in HR were found between the control group and patients who did not use $\beta$-blockers (Table 3 ). Exercise time was the sum of time spent in the test plus recovery time. Mean exercise time was 10.10 min for the control group compared to 8.44 min for the group with Marfan syndrome $(P=0.01$; Table

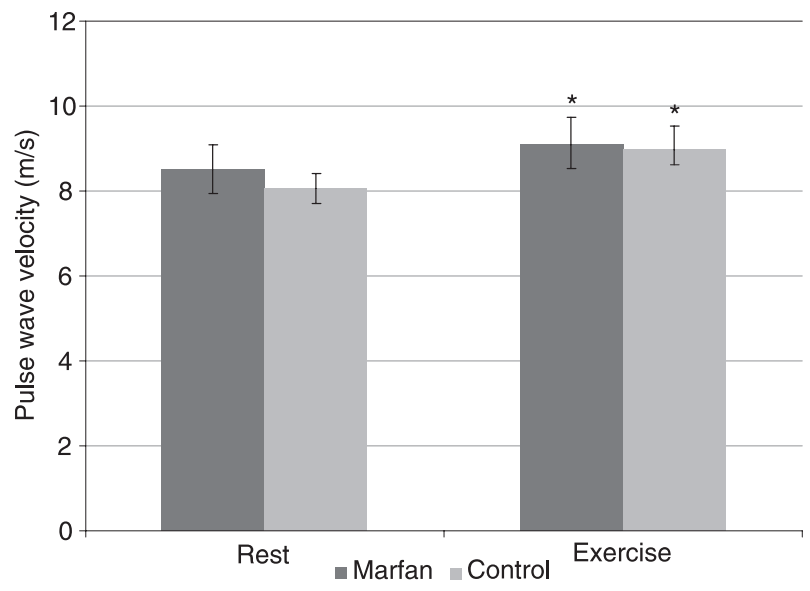

Figure 1. Pulse wave velocity values at rest and at the end of exercise for the patients with Marfan syndrome $(\mathrm{N}=33)$ and for the control group $(\mathrm{N}=18)$. Data are reported as absolute values. ${ }^{*} \mathrm{P}<0.05$ compared to rest ( $t$-test).

Table 2. Physiological variables at rest, at the end of exercise and during the first minute of recovery.

\begin{tabular}{|c|c|c|c|c|c|c|c|c|c|c|c|c|}
\hline & \multicolumn{4}{|c|}{ Marfan $(\mathrm{N}=33)$} & \multicolumn{4}{|c|}{ Control $(N=18)$} & \multirow[t]{2}{*}{ P pred } & \multirow[t]{2}{*}{ Prest } & \multirow[t]{2}{*}{$P$ final } & \multirow[t]{2}{*}{ Prec 1 min } \\
\hline & Pred $/ \%$ at & Rest & Final & $\operatorname{Rec} 1 \mathrm{~min}$ & Pred $/ \%$ at & Rest & Final & $\operatorname{Rec} 1 \mathrm{~min}$ & & & & \\
\hline HR (bpm) & $176 \pm 8 / 90 \pm 11$ & $72 \pm 11$ & $144 \pm 21$ & $121 \pm 20^{a}$ & $175 \pm 5 / 90 \pm 13$ & $78 \pm 12$ & $160 \pm 24$ & $132 \pm 20$ & $0.34 / 0.44$ & 0.08 & $0.01^{*}$ & 0.10 \\
\hline $\mathrm{SAP}(\mathrm{mmHg})$ & & $105 \pm 11$ & $143 \pm 19$ & $125 \pm 16^{c}$ & & $110 \pm 13$ & $157 \pm 21$ & $136 \pm 18$ & & 0.16 & $0.02^{*}$ & \\
\hline $\mathrm{DAP}(\mathrm{mmHg})$ & & $72 \pm 10$ & $82 \pm 13$ & $74 \pm 10^{c}$ & & $69 \pm 8$ & $81 \pm 6$ & $71 \pm 7$ & & 0.33 & 0.24 & \\
\hline Lactate $(\mathrm{mmol} / \mathrm{L})$ & & $3.1 \pm 0.9$ & $6.4 \pm 3.0^{\mathrm{b}}$ & & & $3.3 \pm 1.1$ & $7.3 \pm 2.7$ & & & 0.45 & 0.67 & \\
\hline Ex. time (min) & & & $8.44 \pm 2.24^{b}$ & & & & $10.10 \pm 2.26$ & & & 0.01 & & \\
\hline
\end{tabular}

Data are reported as means $\pm \mathrm{SD}$. $\mathrm{HR}=$ heart rate; $\mathrm{SAP}=$ systolic blood pressure; $\mathrm{DAP}=$ diastolic blood pressure; Ex. time $=$ exercise time; Pred $/ \%$ at $=$ predicted heart rate $/ \%$ of predicted heart rate attained; Rec 1 min $=$ heart rate 1 min recovery. ${ }^{a} \mathrm{~N}=28 ;{ }^{b} \mathrm{~N}=32 ;{ }^{\mathrm{C}} \mathrm{N}=$ 29. *P $\leq 0.05$ compared to control group ( $t$-test). 
Table 3. Heart rate values for the control group and for patients with Marfan syndrome taking or not $\beta$-blockers.

\begin{tabular}{lccc}
\hline & \multicolumn{3}{c}{ Heart rate $(\mathrm{bpm})$} \\
\cline { 2 - 4 } & $\begin{array}{c}\text { Control } \\
(\mathrm{N}=18)\end{array}$ & $\begin{array}{c}\text { No } \beta \text {-blockers } \\
(\mathrm{N}=16)\end{array}$ & $\begin{array}{c}\text { With } \beta \text {-blockers } \\
(\mathrm{N}=17)\end{array}$ \\
\hline Rest & $78 \pm 12$ & $75 \pm 10$ & $69 \pm 11^{*}$ \\
End of exercise & $160 \pm 24$ & $150 \pm 20$ & $139 \pm 21^{*}$ \\
Recovery & $132 \pm 20$ & $127 \pm 20^{\mathbf{b}}$ & $114 \pm 1 \mathbf{a}^{*}$ \\
\hline
\end{tabular}

Data are reported as means $\pm S D$. ${ }^{a} \mathrm{~N}=13 ;{ }^{b} \mathrm{~N}=15 .{ }^{*} \mathrm{P} \leq 0.05$ compared to control group ( $t$-test).

2). No significant difference was found between groups regarding blood lactate values while at rest or at the end of exercise (Table 2).

\section{Systolic and diastolic arterial pressure}

There were no significant differences between the group with Marfan syndrome and the control group regarding resting systolic and diastolic arterial pressure values $(P=$ 0.16 and $P=0.34)$. Systolic arterial pressure at the end of the exercise was $157 \pm 21 \mathrm{mmHg}$ in the control group and $143 \pm 19 \mathrm{mmHg}$ in the group with Marfan syndrome, demonstrating a statistically significant difference between groups $(P=0.02$; Table 2$)$. There was no statistically significant difference between groups regarding diastolic arterial pressure at the end of the exercise.

\section{Discussion}

The present study demonstrated that individuals with Marfan syndrome who have either a normal aorta or mild aortic dilatation develop degrees of PWV similar to those of healthy individuals when performing acute exercise of moderate intensity. The physiological response patterns during exercise were maintained in both groups, even when taking into consideration that approximately half of the individuals with Marfan syndrome used $\beta$-blockers. Marfan syndrome patients using $\beta$-blockers had significantly lower HR values at the end of exercise compared to the control group.

Aortic disease is one of the main complications and the major cause of death in patients with Marfan syndrome (17). The analysis of PWV has been proposed to be an adequate tool for assessing arterial distensibility in healthy individuals and those with diseases that affect the cardiovascular system $(18,19)$. Using a transesophageal echocardiogram with patients at rest, Franke et al. (20) found lesser distensibility in asymptomatic patients with Marfan syndrome when compared to healthy individuals.

The analysis of resting PWV in the present study revealed no statistically significant differences between the group of patients with Marfan syndrome and the control group. This suggests a similar distensibility pattern between the two populations when at rest, even in cases of patients with mild aortic dilatation. However, the numerical results of the present study cannot be compared directly to those reported by Franke et al. (20) since the methods for analysis were different.

The PWV values obtained from measurements taken immediately after patients had lain down (in order to provide adequate quality for the measurements) at the end of exercise were elevated in both groups when compared to resting values. This suggests an acute increase in arterial stiffness with the exertion performed and demonstrates that the established exercise was sufficient to cause changes in arterial elasticity. Analyzing the acute effect of isometric grip strength exercises, Lydakis et al. (21) found a reduction in the transition time of the pulse wave, thereby suggesting an increase in the stiffness of central arteries. Other publications have also reported an increase in PWV and aortic stiffness in normal sedentary individuals and athletes performing exercises (22-24). However, the increase in blood pressure during exercise is a factor interfering with the variation of PWV and aortic distensibility, since the increase in pulse pressure leads to a greater demand on the elastin/collagen structure of the central arteries (25). Furthermore, the increase in wave velocity found in the presence of an increase in arterial stiffness results in a premature return of the reflection wave while still in the systolic phase, thereby producing an increase in systolic arterial pressure (26). In the present study, there was an increase in systolic arterial pressure at the end of exercise and the maintenance of diastolic arterial pressure, with a consequent variation in pulse pressure, which is in agreement with the aforementioned findings. Analysis of covariance of the adjustment in PWV together with the final systolic arterial pressure revealed changes in this variable when the patients of both groups were at rest and at the end of exertion similar to that for PWV alone.

The comparison of resting and final PWV values of patients with Marfan syndrome who used $\beta$-blockers and patients with Marfan syndrome who did not use this medication revealed no statistically significant differences between the two subgroups.

We believe that the PWV values were not different from the control group, not even when at rest, because the aortic alterations in this population were either not yet sufficient to affect the distensibility or stiffness of the aorta or were suppressed by the use of $\beta$-blockers.

The chronotropic response and systolic arterial pressure of the patients with Marfan syndrome in relation to the submaximal effort employed proved to be slightly reduced when compared to that of the control group. This finding may have occurred because of a poorer degree of cardiovascular conditioning, which is corroborated by the shorter total exercise time achieved by the group with Marfan 
syndrome. Furthermore, these patients are instructed by their cardiologists not to perform any type of moderate or intense physical activity, which may also have influenced this finding due to the fact that they have a more sedentary lifestyle (27). We should also consider that 17 patients used $\beta$-blockers and had their predicted HR adjusted. However, no statistical differences were found in resting or final HR between patients with Marfan syndrome who used this medication and those who did not. Phelps et al. (28) found considerable variability in the pharmacokinetics of atenolol in patients with and without Marfan syndrome, which may have influenced the results of the present study.

Analysis of the increase in blood lactate revealed no statistically significant differences between the Marfan syndrome and control groups. However, the levels found may suggest that the participants had reached higher than expected degrees of exertion, inferring a greater cardiovascular load (15). This increase in lactate also corroborates the fact that the effort made was effective and caused metabolic alterations.

A limitation of the present study may have been the time for determining the curves as soon as the patient had returned to bed. Due to the increased HR, the machine often selected two curves at the same time, which prevented immediate acceptance by the system and resulted in a new acquisition command. Moreover, the instrument used for the evaluation of PWV may not have had sufficient sensitivity to identify alterations in the distensibility and stiffness of the aorta expected in this population. This point may suggest that PWV is not a good method for the analysis of acute aortic distensibility even with the partial correction by ANCOVA. Furthermore, even these corrections of PWV may still not be considered by some as a good measure to assess acute changes in vascular compliance. The number of patients with Marfan syndrome able to perform the exercise, together with the exclusion criteria, limited the number of participants. Likewise, it is currently difficult to encounter patients with Marfan syndrome who do not use $\beta$-blockers, which is why we chose to include patients who took this medication. This decision also provided the

\section{References}

1. De Paepe A, Devereux RB, Dietz HC, Hennekam RC, Pyeritz RE. Revised diagnostic criteria for the Marfan syndrome. Am J Med Genet 1996; 62: 417-426.

2. Dean JC. Management of Marfan syndrome. Heart 2002; 88 : 97-103.

3. Bramwell JC, Hill AV. Velocity of transmission of the pulsewave and elasticity of arteries. Lancet 1922; 1: 891-892.

4. Ferrier KE, Waddell TK, Gatzka CD, Cameron JD, Dart AM, Kingwell BA. Aerobic exercise training does not modify large-artery compliance in isolated systolic hypertension. Hypertension 2001; 38: 222-226.

5. Trzos E, Kurpesa M, Rechcinski T, Wierzbowska-Drabik opportunity to compare findings between patients with Marfan syndrome with and without the use of $\beta$-blockers as an initial hypothesis of the study. Another limitation was that the load control on the stationary bike did not allow direct adjustments, which, in turn, did allow an analysis of HR levels and loads.

The present study demonstrates that patients with Marfan syndrome and either a normal aorta or mild aortic dilatation (as assessed by an echocardiogram) exhibit aortic distensibility similar to that of sedentary control individuals when performing submaximal exercise. However, these patients exhibited lower chronotropic and blood pressure responses than controls, possibly due to a lesser degree of physical effort in daily activities (with a consequent lack of physical conditioning), along with the use of $\beta$-blockers. Analysis of the results demonstrates that the immediate effects of moderate-intensity exercise on individuals with Marfan syndrome did not lead to different results from those achieved by the control population, especially among patients who did not use $\beta$-blockers. The beneficial effects of a regular physical exercise program without pronounced loads or risk of bodily collision may produce cardiovascular adaptations that favor the quality of life of these individuals. An analysis of the results obtained here and those encountered in the literature leads us to believe in the protective role therapeutic exercise may provide to these individuals. However, further studies should utilize more sophisticated methods and equipment to be adequately validated and should be carried out employing adequate exercise as a complementary therapy in the treatment of Marfan syndrome. No complications were found during this protocol in Marfan patients.

\section{Acknowledgments}

The authors would like to thank the participants of the present study and all members of the Cardiology Sector of Universidade Federal de São Paulo who either directly or indirectly assisted in the present study. P. Peres received a grant from the Brazilian financing agency CNPq.
$\mathrm{K}$, Krzeminska-Pakula M. The influence of physical rehabilitation on arterial compliance in patients after myocardial infarction. Cardiol J 2007; 14: 366-371.

6. Jondeau G, Boutouyrie P, Lacolley P, Laloux B, Dubourg $\mathrm{O}$, Bourdarias JP, et al. Central pulse pressure is a major determinant of ascending aorta dilation in Marfan syndrome. Circulation 1999; 99: 2677-2681.

7. Hirata K, Triposkiadis F, Sparks E, Bowen J, Wooley CF, Boudoulas H. The Marfan syndrome: abnormal aortic elastic properties. J Am Coll Cardiol 1991; 18: 57-63.

8. Braverman AC. Exercise and the Marfan syndrome. Med Sci Sports Exerc 1998; 30: S387-S395. 
9. Maron BJ, Ackerman MJ, Nishimura RA, Pyeritz RE, Towbin JA, Udelson JE. Task Force 4: HCM and other cardiomyopathies, mitral valve prolapse, myocarditis, and Marfan syndrome. J Am Coll Cardiol 2005; 45: 1340-1345.

10. Schvartzman PR, Fuchs FD, Mello AG, Coli M, Schvartzman M, Moreira LB. Normal values of echocardiographic measurements. A population-based study. Arq Bras Cardiol 2000; 75: 107-114.

11. Lopez VM, Perez AB, Moises VA, Gomes L, Pedreira PS, Silva CC, et al. [Serial clinical and echocardiographic evaluation in children with Marfan syndrome]. Arq Bras Cardiol 2005; 85: 314-318.

12. Asmar R, Benetos A, Topouchian J, Laurent P, Pannier B, Brisac AM, et al. Assessment of arterial distensibility by automatic pulse wave velocity measurement. Validation and clinical application studies. Hypertension 1995; 26: 485490.

13. Dabela MLG, Izar MCO, Relvas WGM, Santos AO, Helfenstein T, Monteiro CMC, et al. Different impact of coronary risk factors on pulse wave velocity. Int $J$ Atheroscler 2007; 2: $75-81$

14. Sociedade Brasileira de Cardiologia. I Consenso Nacional de Reabilitação Cardiovascular. http://publicacoes.cardiol. br/consenso/1997/6904/69040010.pdf. Accessed July 11, 2008.

15. Giske L, Stanghelle JK, Rand-Hendrikssen S, Strom V, Wilhelmsen JE, Roe C. Pulmonary function, working capacity and strength in young adults with Marfan syndrome. $J$ Rehabil Med 2003; 35: 221-228.

16. Sociedade Brasileira de Cardiologia. V Diretriz Brasileira de Hipertensão Arterial. http://publicacoes.cardiol.br/consenso/2006/VDiretriz-HA.pdf. Accessed July 11, 2008.

17. Milewicz DM, Dietz HC, Miller DC. Treatment of aortic disease in patients with Marfan syndrome. Circulation 2005; 111: e150-e157.

18. Lehmann ED. Pulse wave velocity as a marker of vascular disease. Lancet 1996; 348: 744.

19. Asmar RA. Alterations of pulse wave velocity in clinical conditions. In: Anonymous, Arterial stiffness and pulse wave velocity - clinical applications. France: Ed. Elsevier; 1999. p 89-134.

20. Franke A, Muhler EG, Klues HG, Peters K, Lepper W, von Bernuth $\mathrm{G}$, et al. Detection of abnormal aortic elastic properties in asymptomatic patients with Marfan syndrome by combined transoesophageal echocardiography and acoustic quantification. Heart 1996; 75: 307-311.

21. Lydakis C, Momen A, Blaha C, Herr M, Leuenberger UA, Sinoway LI. Changes of elastic properties of central arteries during acute static exercise and lower body negative pressure. Eur J Appl Physiol 2008; 102: 633-641.

22. Asmar RA. Pulse wave velocity and therapy. In: Anonymous, Arterial stiffness and pulse wave velocity - clinical applications. France: Ed. Elsevier; 1999. p 143-159.

23. Sharman JE, McEniery CM, Campbell RI, Coombes JS, Wilkinson IB, Cockcroft JR. The effect of exercise on large artery haemodynamics in healthy young men. Eur J Clin Invest 2005; 35: 738-744.

24. Naka KK, Tweddel AC, Parthimos D, Henderson A, Goodfellow J, Frenneaux MP. Arterial distensibility: acute changes following dynamic exercise in normal subjects. Am J Physiol Heart Circ Physiol 2003; 284: H970-H978.

25. Armentano RL, Levenson J, Barra JG, Fischer El, Breitbart GJ, Pichel RH, et al. Assessment of elastin and collagen contribution to aortic elasticity in conscious dogs. Am J Physiol 1991; 260: H1870-H1877.

26. Vlachopoulos C, Aznaouridis K, Stefanadis C. Clinical appraisal of arterial stiffness: the Argonauts in front of the Golden Fleece. Heart 2006; 92: 1544-1550.

27. Maron BJ, Chaitman BR, Ackerman MJ, Bayes de LA, Corrado D, Crosson JE, et al. Recommendations for physical activity and recreational sports participation for young patients with genetic cardiovascular diseases. Circulation 2004; 109: 2807-2816.

28. Phelps SJ, Alpert BS, Ward JL, Pieper JA, Lima JJ. Absorption pharmacokinetics of atenolol in patients with the Marfan syndrome. J Clin Pharmacol 1995; 35: 268-274. 